\title{
Association of teriparatide adherence and persistence with clinical and economic outcomes in Medicare Part $D$ recipients: a retrospective cohort study
}

\author{
Leslie Hazel-Fernandez ${ }^{1 *}$, Anthony M Louder', Shonda A Foster ${ }^{2}$, Claudia L Uribe ${ }^{1}$ and Russel T Burge ${ }^{2}$
}

\begin{abstract}
Background: Improper medication adherence is associated with increased morbidity, healthcare costs, and fracture risk among patients with osteoporosis. The objective of this study was to evaluate the healthcare utilization patterns of Medicare Part D beneficiaries newly initiating teriparatide, and to assess the association of medication adherence and persistence with bone fracture.

Methods: This retrospective cohort study assessed medical and pharmacy claims of 761 Medicare members initiating teriparatide in 2008 and 2009. Baseline characteristics, healthcare use, and healthcare costs 12 and 24 months after teriparatide initiation, were summarized. Adherence, measured by Proportion of Days Covered (PDC), was categorized as high (PDC $\geq 80 \%)$, moderate $(50 \% \geq$ PDC $<80 \%)$, and low (PDC $<50 \%)$. Non-persistence was measured as refill gaps in subsequent claims longer than 60 days plus the days of supply from the previous claim. Multivariate logistic regression evaluated the association of adherence and persistence with fracture rates at 12 months.

Results: Within 12 months of teriparatide initiation, 21\% of the cohort was highly-adherent. Low-adherent or non-persistent patients visited the ER more frequently than did their highly-adherent or persistent counterparts $(x 2=5.01, p<0.05$ and $x 2=5.84, p<0.05)$, and had significantly lower mean pharmacy costs $(\$ 4,361$ versus $\$ 13,472$ and $\$ 4,757$ versus $\$ 13,187, \mathrm{p}<0.0001)$. Furthermore, non-persistent patients had significantly lower total healthcare costs. The healthcare costs of highly-adherent patients were largely pharmacy-related. Similar patterns were observed in the 222 patients who had fractures at 12 months, among whom 89\% of fracture-related costs were pharmacy-related. The regression models demonstrated no significant association of adherence or persistence with 12-month fractures. Six months before initiating teriparatide, 50.7\% of the cohort had experienced at least 1 fracture episode. At 12 months, these patients were nearly 3 times more likely to have a fracture (OR=2.9, 95\% C.I. 2.1-4.1 $\mathrm{p}<0.0001)$.
\end{abstract}

Conclusions: Adherence to teriparatide therapy was suboptimal. Increased pharmacy costs seemed to drive greater costs among highly-adherent patients, whereas lower adherence correlated to greater ER utilization but not to greater costs. Having a fracture in the 6 months before teriparatide initiation increased fracture risk at follow-up.

Keywords: Osteoporosis, Teriparatide, Adherence, Persistence, Discontinuation, Medicare part D, Fractures, Outcomes

\footnotetext{
* Correspondence: Ihazel-fernandez@humana.com

${ }^{1}$ Competitive Health Analytics Inc., Humana Inc, 3501 SW 160 Ave, Miramar,

FL 33029, USA

Full list of author information is available at the end of the article
} 


\section{Background}

Osteoporosis is a breakdown and weakening of the bones that affects over 10 million individuals in the U.S. and is linked to increased morbidity, mortality, and healthcare costs $[1,2]$. Effective medications have been developed to treat osteoporosis, but adherence to treatment is suboptimal [3-5]. Proper adherence may delay and prevent fracture, future bone loss, and other negative health outcomes [6,7]. After one year of therapy, many patients discontinue or decrease dosages of daily oral and injectable medications [7]; this non-persistence and underuse may reduce the drugs' effectiveness, placing patients at increased risk of fractures and other negative outcomes $[1,4,7]$.

Adherence and persistence with prescribed medication are extremely important for patients at high risk for fractures since these patients are at a much greater risk for fractures relative to patients with mild to moderate osteoporosis. Available studies suggest that reasons for patients' suboptimal adherence or persistence with osteoporosis medications include concerns about side effects, inconvenience of drug regimens, and drug costs $[8,9]$. Drug cost concerns are of greater relevance to patients who are prescribed injectable medications because these medications tend to be more costly than oral agents. A recent prospective observational study of patients taking teriparatide, a daily injectable drug developed to treat patients who are at high risk for fractures or for whom first-line treatments have been ineffective [10], found that patients who discontinued therapy within 12 months did so primarily due to problems with paying for prescriptions and perceptions that the benefits of the treatment were outweighed by their concerns about the treatment [11].

The cost sharing attributes of the Medicare Part D prescription drug plan may pose a financial challenge to patients with osteoporosis, especially to those taking costlier medications [12]. Medicare Part D is a part of Medicare, which is a U.S. government-sponsored health insurance that provides coverage primarily for adults over the age of 65. In Medicare Part D, government payments for drug purchases stop when a beneficiary reaches an annual spending limit. Thereafter, beneficiaries must pay for $100 \%$ of their prescription costs until their out-of-pocket costs reach a pre-specified threshold (catastrophic coverage) [12,13]. Patients taking costly medications for chronic conditions such as osteoporosis may reach this spending limit sooner, and as a consequence, they may resort to cost-coping behaviors to manage their healthcare spending during the resulting gaps in coverage [14-17]. Some studies of Medicare Part $\mathrm{D}$ patients have identified cost-coping strategies such as using medications less frequently than prescribed, discontinuing medications, not filling prescriptions, and switching to less expensive agents [18-20]. Other studies suggest that cost-related responses are more common among patients who have better knowledge of their benefits and who report fewer financial burdens [21,22]. With regard to teriparatide use among Medicare beneficiaries, one study posits that patients' experiences with the drug affect their healthcare spending habits and their adherence during gaps in coverage [23].

In a recent retrospective cohort study, Tamariz and colleagues evaluated patient persistence with teriparatide and other osteoporosis medications as compared to persistence with biologic therapies used to treat rheumatoid arthritis (RA) and multiple sclerosis (MS) [12]. The authors compared the pharmacy claims of health plan members who had Medicare Part D prescription plans with and without coverage gaps. The findings indicated that patients taking osteoporosis medications, particularly teriparatide, were more likely to discontinue taking their medication if they reached the coverage gap, whereas no such association was observed for patients taking medications to treat RA or MS. A limitation of this study was that it did not assess the medical and economic outcomes associated with these persistence patterns. In another retrospective cohort study, $\mathrm{Yu}$ and colleagues evaluated the pharmacy and medical claims of new teriparatide users aged 18 years and older [6]. The authors found that patients' optimal adherence was associated with a reduced risk of fractures at 6, 12 and 18 months following teriparatide initiation. This study did not specifically assess fracture outcomes among Medicare part D beneficiaries [6].

In order to address gaps noted in previous research, the present study evaluated outcomes associated with adherence and persistence among Medicare Part D beneficiaries. This retrospective investigation was conducted on a cohort of Medicare Advantage with Prescription Drug coverage (MAPD) members who had initiated prescriptions for teriparatide in 2008 and 2009 and who were enrolled through a large national healthcare provider. The study evaluated the outcomes of fracture rates, inpatient/outpatient resource utilization, and direct medical costs.

\section{Results}

\section{Sample selection}

Figure 1 depicts the attrition diagram which identifies how many health plan members were removed from the sample due to the specified exclusion/inclusion criteria. A total of 2,688 health plan members were enrolled in the Medicare Advantage Plan between January 1, 2008 and December 31, 2009 and were identified as receiving a prescription for teriparatide. Most of those identified were excluded because they were not naïve to teriparatide during the identification period, were not continuously 


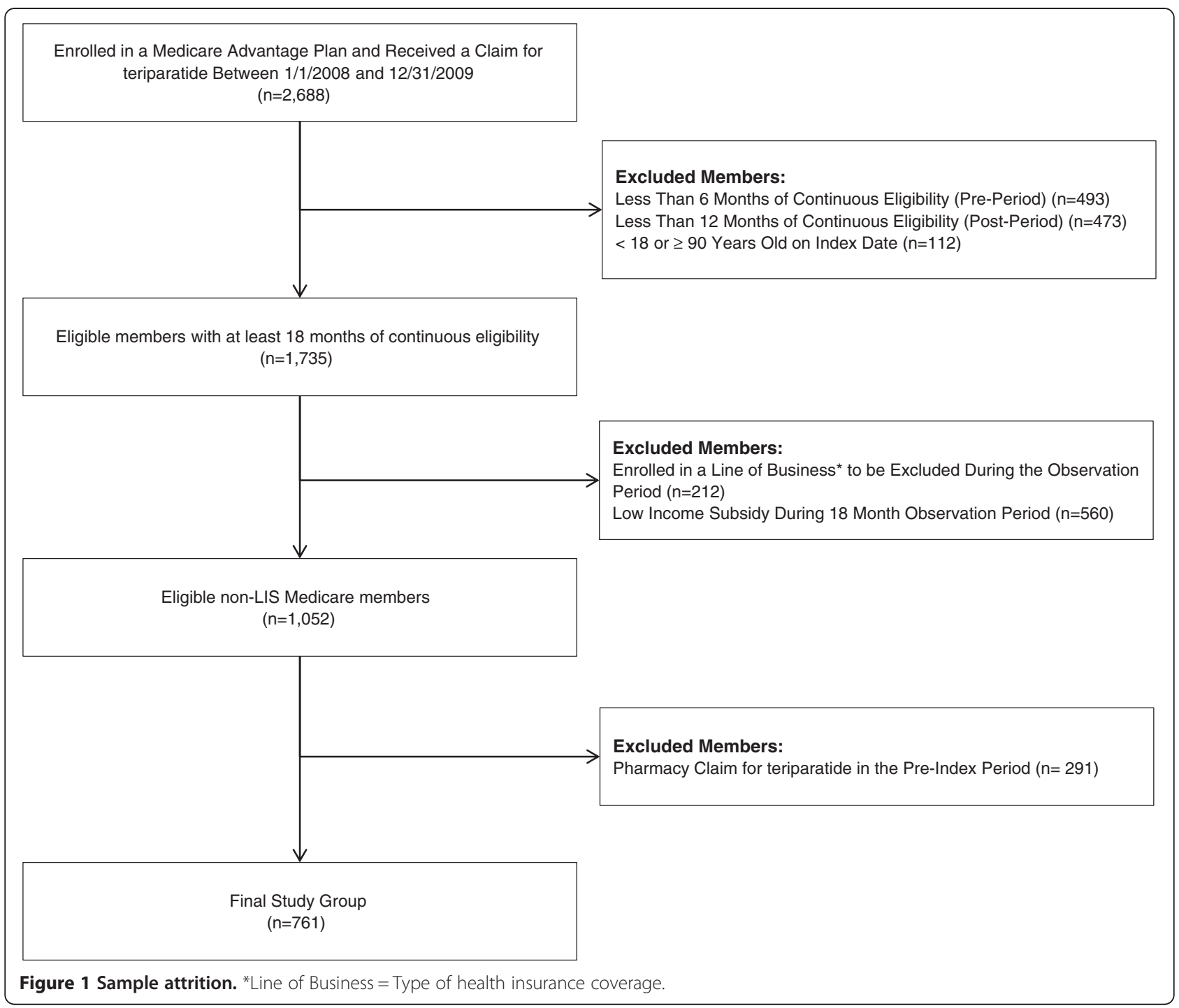

enrolled for at least 6 months pre-index and at least 12 months post index, and/or were eligible for the low income subsidy (LIS) benefit program. The Final Study Group was comprised of 761 members, $36 \%$ of which (272 members) had 24 months of continuous post-period eligibility.

\section{Patient characteristics}

Table 1 depicts the baseline demographic characteristics of the final sample $(\mathrm{n}=761)$. The average age of patients was $73 \pm 8.4$ years, and approximately $66 \%$ of the sample was 70 years of age or older. Of the total sample, $88.7 \%$ were female, and $93.4 \%$ were Caucasian. Participants were primarily from the Southern and Midwestern United States (approximately 66\% and 21\%, respectively).

Table 2 summarizes the clinical characteristics of the patient cohort and the percentages of patients who reached the Medicare Part D coverage gap in the 12 months following initiation of teriparatide. At baseline, the Deyo-Charlson score for the group was relatively low (mean of 1.1) indicating a low level of significant comorbidities. The top three most prevalent comorbidities were other disorders of bone and cartilage (ICD-9 code 733.xx; 98.8\%), essential hypertension (ICD-9 code 401.xx; 71.7\%), and disorders of lipid metabolism (ICD-9 code 272.xx; 69.5\%). With regard to the use of osteoporosis drug therapy in the 6 month 'pre' period, $46.4 \%$ of the cohort (353 patients) had used an osteoporosis medication. Among these users, the most frequently used medications were bisphosphonates (80\%), calcitonin (11\%), selective estrogen receptor modulator (11\%), and conjugated estrogen (6\%). With respect to prior fractures, $50.7 \%$ of the sample (386 patients) had experienced a fracture during the 6 months before initiation of teriparatide. In the 12-months following initiation of teriparatide, $77 \%$ of the sample (586 patients) had experienced a gap in Medicare Part D prescription drug coverage. 
Table 1 Demographic characteristics

\begin{tabular}{lc}
\hline Characteristic & Count \\
\hline Total Patients & 761 \\
Age, years (Mean, Standard Deviation) & $73.3( \pm 8.4)$ \\
Age Distribution (Number, Percent) & \\
$18-29$ & 0 \\
$30-39$ & 0 \\
$40-49$ & $6(0.8 \%)$ \\
$50-59$ & $31(4.1 \%)$ \\
$60-69$ & $223(29.3 \%)$ \\
$70-79$ & $316(41.5 \%)$ \\
$80-89$ & $185(24.3 \%)$ \\
Female (Number, Percent) & $675(88.7 \%)$ \\
Ethnicity (Number, Percent) & \\
White & $711(93.4 \%)$ \\
Black & $23(3.0 \%)$ \\
Hispanic & $8(1.1 \%)$ \\
Other & $19(2.5 \%)$ \\
Geographic Region (Number, Percent) & \\
Northeast & $17(2.2 \%)$ \\
Midwest & $160(21 \%)$ \\
South & $502(66 \%)$ \\
West & $82(10.8 \%)$ \\
\hline
\end{tabular}

\section{Healthcare resource utilization and costs in the follow-up period}

Overall resource utilization in the 12 and 24-month period following teriparatide initiation is summarized in Table 3. In the 12-month post period, approximately $21 \%$ of the study group had at least 1 hospitalization, $31 \%$ had at least one emergency room (ER) visit, 99.9\% had at least one outpatient visit, and all members had at least one pharmacy claim. In the subgroup of members with 24 months of post-period follow-up (i.e., members with coverage for months 1-24 following teriparatide initiation; $\mathrm{n}=272$ ), 31\% had at least 1 hospitalization, more than $44 \%$ had ER visits, and all members had at least one outpatient visit and at least one pharmacy claim. As noted in Table 3, the mean total healthcare costs (plan paid and member share combined) amounted to $\$ 17,460$ per member at 12 months follow-up. At 24 months the costs were $\$ 30,292$ per member. Inpatient hospitalization costs were on average $\$ 4,585$ (26\%) at 12 months and $\$ 8,978$ (29\%) at 24 months. Outpatient costs were $\$ 5,713(33 \%)$ at 12 months and $\$ 9,673(32 \%)$ at 24 months. Pharmacy costs allocated to the average member amounted to $\$ 6,762$ (39\%) at 12 months and $\$ 10,787$ (36\%) at 24 months.

Fracture-related costs (plan cost and member share) are summarized in Table 4 for members identified as having a fracture during the 12 - or 24-month post periods (222 and 134 members, respectively). The mean total fracture-related direct costs were $\$ 6,198$ per member at 12 months (i.e., months 1 to 12 ), and $\$ 8,389$ per member at 24 months of follow-up (i.e., months 1 to $24)$. ER-related costs were, on average, $\$ 35$ at 12 months and almost triple that amount at $\$ 100$ at 24 months. In contrast, inpatient hospitalization costs were, on average, $\$ 1,123$ at 12 months, and were $\$ 967$ at 24 months. Mean outpatient costs were $\$ 829$ at 12 months and $\$ 1,007$ at 24 months. The average osteoporosis medication prescription costs allocated to each member amounted to $\$ 3,965$ at 12 months and $\$ 6,061$ at 24 months. Pharmacy costs for acute fracture treatment (pain medication) were close at both time periods at $\$ 246$ per member at 12 months, and $\$ 253$ per member at 24 months.

\section{Description of teriparatide adherence, persistence and economic outcomes}

In order to describe the manner in which adherence and persistence with teriparatide therapy were related to economic outcomes, patients were grouped according to their adherence to the drug during the 12-month follow-up period, and whether they persisted with therapy during the same time frame (see Table 5). Adherence status was reported as high (PDC $\geq 80 \%)$, moderate $(50 \% \leq \mathrm{PDC}<$ $80 \%$ ), and low (PDC $<50 \%)$. Persistence with teriparatide was described as either 'non-persistent' or 'persistent' with treatment. For each of the defined groups, the overall and fracture-related utilization and costs were calculated. Chi Square analyses, ANOVA pairwise comparisons, and $\mathrm{t}$-tests were used to assess significant differences in healthcare utilization and costs depending on patients' adherence or persistence groupings.

\section{Teriparatide adherence/persistence and fracture-related resource utilization - one year post index}

The results in Table 5 show that of the entire cohort, $21 \%$ (163 patients) were highly adherent (PDC $\geq 80 \%$ ) to their teriparatide therapy, and 24\% (181 patients) persisted with taking the medication during the 12 month follow-up period. In addition, the results indicate that for the entire sample, there were significant differences in patients' ER visits, total healthcare costs, and pharmacy costs depending on patients' adherence or persistence. With regard to healthcare resource utilization, Chi Square test results revealed that there were significant differences in the number of members with ER visits depending on how adherent members were or whether or not they were persistent with therapy. Patients who fell into the low-adherence category $(\mathrm{PDC}<50)$ were more likely to have ER visits than were those who demonstrated high adherence (PDC $\geq 80), \quad\left(X^{2}=5.10\right.$, $\mathrm{p}<0.05)$. Similarly, members who were not persistent 
Table 2 Clinical characteristics

\begin{tabular}{|c|c|}
\hline Characteristic & Count \\
\hline Total Patients & 761 \\
\hline \multicolumn{2}{|l|}{ Baseline Clinical Characteristics } \\
\hline \multirow[t]{2}{*}{ Charlson Comorbidity Index* } & $1.1( \pm 1.6)$ \\
\hline & $1[0-12]$ \\
\hline Osteoporosis Drug Utilization in Pre-Period n,(\%) & $353(46.4 \%)$ \\
\hline Bisphosphonate n,(\%) & $283(80.2 \%)$ \\
\hline Calcitonin $n,(\%)$ & $39(11.0 \%)$ \\
\hline Selective Estrogen Receptor Modulator (SERM) n,(\%) & $42(11.9 \%)$ \\
\hline Conjugated Estrogen n,(\%) & $22(6.2 \%)$ \\
\hline Fracture in the 6 Month Pre-Period n, (\%) & $386(50.7 \%)$ \\
\hline Fracture in the 12 Month Pre-Period $n,(\%) * *$ & $443(58.2 \%)$ \\
\hline \multicolumn{2}{|l|}{ Comorbidity (Top 10 Identified) n, (\%) ${ }^{\dagger}$} \\
\hline Other disorders of bone and cartilage (733.) & $752(98.8 \%)$ \\
\hline Essential hypertension (401.) & $546(71.7 \%)$ \\
\hline Disorders of lipid metabolism (272.) & $529(69.5 \%)$ \\
\hline General symptoms (780.) & $474(62.3 \%)$ \\
\hline Other and unspecified disorders of back (724.) & $402(52.8 \%)$ \\
\hline Symptoms involving respiratory system and other chest symptoms (786.) & $391(51.4 \%)$ \\
\hline Other and unspecified disorders of joint (719.) & $382(50.2 \%)$ \\
\hline Osteoarthritis and allied disorders (715.) & $361(47.44 \%)$ \\
\hline Other disorders of soft tissues (729.) & $333(43.8 \%)$ \\
\hline Cataract (366.) & $281(36.9 \%)$ \\
\hline \multicolumn{2}{|l|}{ Post-Period Clinical Characteristics } \\
\hline Fracture in the 12 Month Post-Period n,(\%) & $222(29.2 \%)$ \\
\hline \multirow[t]{2}{*}{ Time Between Index Date and First Fracture (Days)* } & $200( \pm 81)$ \\
\hline & $181[91-364]$ \\
\hline \multirow[t]{2}{*}{ Proportion of Days Covered (PDC) Between Index Date and First Fracture* } & $0.46( \pm 0.3)$ \\
\hline & $0.38[0.08-1.0]$ \\
\hline Reached Part D Coverage Gap During 12 Month Follow-up Period n, (\%) & $586(77 \%)$ \\
\hline Out of Pocket Cost for All Prescription Claims During 12 Month Follow-up & $\$ 2,082( \pm \$ 2,189)$ \\
\hline Period* $^{*}$ & $\$ 991[\$ 30-\$ 10,894]$ \\
\hline Out of Pocket Cost for Teriparatide Prescription Claims During 12 Month & $\$ 1,247( \pm \$ 1,832)$ \\
\hline Follow-up Period* & $\$ 228[\$ 15-\$ 7,165]$ \\
\hline
\end{tabular}

*Mean (M), standard deviation (SD), median, [range].

** Data for fracture in 12 month pre-period were not available for the entire cohort.

${ }^{\dagger}$ Based on the first 3 digits of ICD-9 CM code.

were more likely to visit the ER during the study period than were the members who were persistent, $\left(\chi^{2}=5.84\right.$, $\mathrm{p}<0.05)$.

With regard to total healthcare costs, the results in Table 5 suggest differences in costs depending on the adherence or persistence category that a patient belonged to. The results of $t$-tests indicated significant differences in total costs when comparisons were made between members who were not persistent (i.e., discontinued therapy) and those that were persistent. Non-persistent members had significantly lower mean healthcare costs $(M=\$ 16,341$, $\mathrm{SD}=\$ 37,485)$ as compared to those who were persistent $(\mathrm{M}=\$ 21,046 \mathrm{SD}=\$ 13,560, \mathrm{t}=2.54, \mathrm{p}<0.05)$. ANOVA pair-wise comparison revealed that members with PDC $<50$ had lower total healthcare costs as compared to those with $\mathrm{PDC} \geq 80 \%$. The average total costs during the follow up period were lower in members demonstrating low adherence as compared to those with high adherence, 
Table 3 Total healthcare resource utilization and costs

\begin{tabular}{|c|c|c|}
\hline Measure & 12 month post period & 24 month post period \\
\hline Total Patients & 761 & 272 \\
\hline \multicolumn{3}{|l|}{ Inpatient Hospitalization } \\
\hline Members with Hospitalization n,(\%) & $158(20.8 \%)$ & $85(31.3 \%)$ \\
\hline \multirow[t]{2}{*}{ Hospitalizations Per Member** } & $0.4( \pm 0.9)$ & $0.7( \pm 1.4)$ \\
\hline & $0[0-6]$ & $0[0-8]$ \\
\hline \multicolumn{3}{|l|}{ Emergency Room Visits } \\
\hline Members with Visit n,(\%) & $236(31.01 \%)$ & $121(44.49 \%)$ \\
\hline \multirow[t]{2}{*}{ Visits Per Member** } & $0.6( \pm 1.6)$ & $1.3( \pm 3.0)$ \\
\hline & $0[0-30]$ & $0[0-42]$ \\
\hline \multicolumn{3}{|l|}{ Outpatient Visits } \\
\hline Members with Visit n,(\%) & 760 (99.9\%) & $272(100.0 \%)$ \\
\hline \multirow[t]{2}{*}{ Visits Per Member** } & 35. $( \pm 33.5)$ & $68.6( \pm 61.7)$ \\
\hline & $25[0-320]$ & $53[9-698]$ \\
\hline \multicolumn{3}{|l|}{ Pharmacy Claims } \\
\hline Members with Pharmacy Claim, n,(\%) & $761(100.0 \%)$ & $272(100.0 \%)$ \\
\hline \multirow[t]{2}{*}{ Pharmacy Claims Per Member** } & $45.99( \pm 31.9)$ & $98.60( \pm 65.9)$ \\
\hline & $39[1-214]$ & $83[4-366]$ \\
\hline \multirow[t]{2}{*}{ Total Healthcare Cost** } & $\$ 17,460( \pm \$ 33,437)$ & $\$ 30,292( \pm \$ 67,230)$ \\
\hline & $\$ 10,896[\$ 1,097-\$ 758,916]$ & $\$ 18,106[\$ 2,859-\$ 1,060,087]$ \\
\hline \multirow[t]{2}{*}{ Inpatient Hospitalization Cost** } & $\$ 4,585( \pm \$ 23,717)$ & $\$ 8,978( \pm \$ 48,891)$ \\
\hline & $\$ 0[\$ 0-\$ 562,430]$ & $\$ 0[\$ 0-\$ 774,152]$ \\
\hline \multirow[t]{2}{*}{ Emergency Room Visit Cost** } & $\$ 400( \pm \$ 2,433)$ & $\$ 855( \pm \$ 4,312)$ \\
\hline & $\$ 0[\$ 0-\$ 62,129]$ & $\$ 0[\$ 0-\$ 68,565]$ \\
\hline \multirow[t]{2}{*}{ Outpatient Cost** } & $\$ 5,713( \pm \$ 8,397)$ & $\$ 9,673( \pm \$ 12,448)$ \\
\hline & $\$ 2,724[\$ 0-\$ 91,816]$ & $\$ 6,067[\$ 0-\$ 144,264]$ \\
\hline \multirow[t]{2}{*}{ Pharmacy Cost** } & $\$ 6,762( \pm \$ 6,359)$ & $\$ 10,787( \pm \$ 10,996)$ \\
\hline & $\$ 4,253[\$ 829-\$ 72,703]$ & $\$ 6,343[\$ 855-\$ 73,434]$ \\
\hline
\end{tabular}

**Mean, standard deviation, median, [range].

although the differences were not significant $(M=$ $\$ 15,528, \quad \mathrm{SD}=\$ 37,713$ versus $(\mathrm{M}=\$ 21,033, \quad \mathrm{SD}=$ $\$ 13,136, \mathrm{~F}=3.35, \mathrm{p}=0.068)$.

Similar to observations of ER visits and total costs, the results suggested significant differences in pharmacy costs depending on adherence or persistence groupings. The ANOVA pair-wise comparison revealed that the average pharmacy costs of members with low adherence $(\mathrm{M}=$ $\$ 4,361, \mathrm{SD}=\$ 5,344$ ) were significantly lower than the costs of members with high adherence $(M=\$ 13,472$, $\mathrm{SD}=\$ 3,787, \mathrm{~F}=411.35 \mathrm{p}<0.0001)$. In addition, t-test results indicated significant differences in pharmacy costs when comparisons were made between members who were non-persistent and those that were persistent. Non-persistent members had lower pharmacy costs $(\mathrm{M}=\$ 4,757, \mathrm{SD}=\$ 5,597)$ than their persistent counterparts $(M=\$ 13,187, \mathrm{SD}=\$ 3,935, \mathrm{t}=22.57, \mathrm{p}<0.0001)$.

As shown in Table 6, similar adherence/persistence groupings were evaluated for the 222 patients identified as having a fracture during the 12-month follow-up period. Among this group of patients, nearly 18\% (39 patients) were identified as being highly-adherent, and 19\% (43 patients) were identified as being persistent with teriparatide therapy during the 12 month follow-up period. With regard to fracture-related resource utilization and costs, there were significant differences noted in total costs and 
Table 4 Fracture-related healthcare resource utilization and costs ${ }^{\dagger}$

\section{Measure}

Total Patients

Fracture-Related Inpatient Hospitalization

Members with Hospitalization n,(\%)

Hospitalizations Per Member**

Fracture-Related Emergency Room Visits

Members with Visit n,(\%)

Visits Per Member**

Fracture-Related Outpatient Visits

$$
\text { Members with Visit n,(\%) }
$$

Visits Per Member**

Fracture-Related Pharmacy Claims

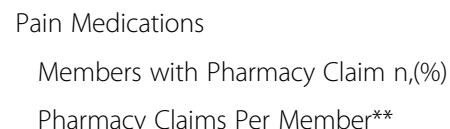

Pharmacy Claims Per Member**

Osteoporosis Medications

Members with Pharmacy Claim n,(\%)*

Pharmacy Claims Per Member**

Total Fracture-Related Cost**

Inpatient Hospitalization Cost**

Emergency Room Visit Cost**

Outpatient Cost**

Osteoporosis Medication Cost**

Pain Medication Cost**
$\$ 6,198( \pm \$ 6,578)$

$\$ 3,260[\$ 854-\$ 40,637]$

$\$ 3,965( \pm \$ 3,640)$

$\$ 2,459[\$ 779-\$ 14,910]$

12 month post period

222

21 (9.5\%)

$0.1( \pm 0.4)$

0 [0 - 2]

35 (15.8\%)

$0.2( \pm 0.5)$

0 [0 - 3]

213 (95.9\%)

$4.1( \pm 7.4)$

2 [0 - 65]

$161(72.5 \%)$

$5.6( \pm 7.7)$

$2[0-43]$

$222(100 \%)$

$5.8( \pm 4.5)$

4 [1 - 18]

$\$ 1,123( \pm \$ 4,506)$

$\$ 0[\$ 0-\$ 37,355]$

$\$ 35( \pm \$ 117)$

\$0 [\$0 - \$753]

$\$ 829( \pm \$ 2,297)$

$\$ 179[\$ 0-\$ 23,383]$

\$246 ( $\pm \$ 758)$

$\$ 24[\$ 0-\$ 6,276]$
24 month post period

134

$11(8.2 \%)$
$0.1( \pm 0.4)$
$0[0-2]$

$33(24.6 \%)$

$0.3( \pm 0.6)$

$0[0-3]$

$126(94 \%)$

$4.6( \pm 8.55)$

2 [0 - 61]

$110(82.1 \%)$

$9.2( \pm 11.9)$

4 [0 - 55]

134 (100\%)

10. $( \pm 8.7)$

$6[1-29]$

$\$ 8,389( \pm \$ 8,650)$

$\$ 3,944[\$ 854-\$ 47,033]$

$\$ 967( \pm \$ 3,582)$

$\$ 0[\$ 0-\$ 22,387]$

$\$ 100( \pm \$ 674)$

$\$ 0[\$ 0-\$ 7,692]$

$\$ 1,007( \pm \$ 3,128)$

$\$ 141[\$ 0-\$ 23,545]$

$\$ 6,061( \pm \$ 6,737)$

$\$ 2,603[\$ 784-\$ 22,965]$

$\$ 253( \pm \$ 605)$

$\$ 35[\$ 0-\$ 3,575]$

${ }^{\dagger}$ Among patients with a fracture.

*All patients with a fracture had an osteoporosis medication claim.

**Mean, standard deviation, median, [range]. 
Table 5 Teriparatide utilization and overall utilization and costs in the $\mathbf{1 2}$ month follow-up period

\begin{tabular}{|c|c|c|c|c|c|}
\hline Measure & $\begin{array}{c}\mathrm{PDC}^{*}<50 \% \\
\text { (low adherent) }\end{array}$ & $\begin{array}{c}\text { PDC } 50 \% \leq 80 \% \\
\text { (moderately } \\
\text { adherent) }\end{array}$ & $\begin{array}{c}\text { PDC } \geq 80 \% \\
\text { (highly adherent) }\end{array}$ & Persistent & $\begin{array}{c}\text { Non-persistent } \\
\text { (60 day gap) }\end{array}$ \\
\hline Total Patients & 541 & 57 & 163 & 181 & 580 \\
\hline \multicolumn{6}{|l|}{ Inpatient Hospitalizations } \\
\hline $\begin{array}{l}\text { Members with } \\
\text { Hospitalization } n,(\%)\end{array}$ & $116(21.4 \%)$ & $16(28.1 \%)$ & $26(16 . \%)$ & $31(17.13 \%)$ & $127(21.9 \%)$ \\
\hline \multirow{2}{*}{$\begin{array}{l}\text { Hospitalizations Per } \\
\text { Member** }\end{array}$} & $0.4( \pm 0.9)$ & $0.5( \pm 1.10)$ & $0.2( \pm 0.6)$ & $0.3( \pm 0.7)$ & $0.4( \pm 0.9)$ \\
\hline & $0[0-6]$ & $0[0-5]$ & $0[0-4]$ & $0[0-4]$ & $0[0-6]$ \\
\hline \multicolumn{6}{|l|}{ Emergency Room Visits } \\
\hline Members with Visit $\mathrm{n},(\%) \Delta ¥$ & $180(33.27 \%)$ & $17(29.82 \%)$ & $39(23.93 \%)$ & $43(23.76 \%)$ & $193(33.28 \%)$ \\
\hline \multirow[t]{2}{*}{ Visits Per Member** } & $0.7( \pm 1.8)$ & $0.5( \pm 0.8)$ & $0.4( \pm 1.1)$ & $0.4( \pm 1.04)$ & $0.7( \pm 1.7)$ \\
\hline & $0[0-30]$ & $0[0-3]$ & $0[0-9]$ & $0[0-9]$ & $0[0-30]$ \\
\hline \multicolumn{6}{|l|}{ Outpatient Visits } \\
\hline Members with Visit $n,(\%)$ & $540(99.8 \%)$ & $57(100.0 \%)$ & $163(100.0 \%)$ & $181(100.0 \%)$ & $579(99.8 \%)$ \\
\hline \multirow[t]{2}{*}{ Visits Per Member** } & $35.4( \pm 34.2)$ & 39. $( \pm 39.8)$ & $32.3( \pm 28.6)$ & $32.7( \pm 29.7)$ & $35.7( \pm 34.6)$ \\
\hline & $26[0-320]$ & $24[1-192]$ & $23[3-195]$ & $23[3-195]$ & $26[0-320]$ \\
\hline \multicolumn{6}{|l|}{ Pharmacy Claims } \\
\hline $\begin{array}{l}\text { Members with Pharmacy } \\
\text { Claim } n,(\%)\end{array}$ & $541(100.0 \%)$ & $57(100.0 \%)$ & $163(100.0 \%)$ & $181(100.0 \%)$ & $580(100.0 \%)$ \\
\hline \multirow{2}{*}{$\begin{array}{l}\text { Pharmacy Claims Per } \\
\text { Member** }^{* *}\end{array}$} & $44.36( \pm 31.01)$ & $43.96( \pm 28.70)$ & $52.12( \pm 35.06)$ & $51.20( \pm 34.72)$ & $44.37( \pm 30.79)$ \\
\hline & $38[1-214]$ & $37[4-127]$ & $41[6-186]$ & $40[6-186]$ & $39[1-214]$ \\
\hline \multirow[t]{2}{*}{ Total Healthcare Cost ${ }^{* *} \dagger \neq$} & $\$ 15,528( \pm \$ 37,713)$ & $\$ 25,574( \pm \$ 28,620)$ & $\$ 21,033( \pm \$ 13,136)$ & $\$ 21,046( \pm \$ 13,560)$ & $\$ 16,341( \pm \$ 37,485)$ \\
\hline & $\begin{array}{l}\$ 7,272[\$ 1,097- \\
\quad \$ 758,916]\end{array}$ & $\begin{array}{l}\$ 15,124[\$ 6,403- \\
\quad \$ 172,809]\end{array}$ & $\begin{array}{c}\$ 15,777[\$ 9,745- \\
\$ 87,302]\end{array}$ & $\begin{array}{c}\$ 15,777[\$ 7,901- \\
\$ 87,302]\end{array}$ & $\begin{array}{c}\$ 7,562[\$ 1,097- \\
\$ 758,916]\end{array}$ \\
\hline \multirow{2}{*}{$\begin{array}{l}\text { Inpatient Hospitalization } \\
\text { Cost** }\end{array}$} & $\$ 4,897( \pm \$ 26,941)$ & $\$ 8,448( \pm \$ 21,125)$ & $\$ 2,198( \pm \$ 7,342)$ & $\$ 2,535( \pm \$ 7,961)$ & $\$ 5,224( \pm \$ 26,775)$ \\
\hline & $\$ 0[\$ 0-\$ 562,430]$ & $\$ 0[\$ 0-\$ 123,486]$ & $\$ 0[\$ 0-\$ 57,410]$ & $\$ 0[\$ 0-\$ 57,410]$ & $\$ 0[\$ 0-\$ 562,430]$ \\
\hline \multirow{2}{*}{$\begin{array}{l}\text { Emergency Room Visit } \\
\text { Cost }^{* *}\end{array}$} & $\$ 465( \pm \$ 2,840)$ & $\$ 330( \pm \$ 971)$ & $\$ 211( \pm \$ 711)$ & $\$ 205( \pm \$ 686)$ & $\$ 461( \pm \$ 2,759)$ \\
\hline & $\$ 0[\$ 0-\$ 62,129]$ & $\$ 0[\$ 0-\$ 5,486]$ & $\$ 0[\$ 0-\$ 6,124]$ & $\$ 0[\$ 0-\$ 6,124]$ & $\$ 0[\$ 0-\$ 62,129]$ \\
\hline \multirow[t]{2}{*}{ Outpatient Cost ${ }^{* *}$} & $\$ 5,806( \pm \$ 8,860)$ & $\$ 6,439( \pm \$ 8,880)$ & $\$ 5,151( \pm \$ 6,428)$ & $\$ 5,118( \pm \$ 6,290)$ & $\$ 5,899( \pm \$ 8,950)$ \\
\hline & $\$ 2,689[\$ 0-\$ 91,816]$ & $\$ 2,724[\$ 93-\$ 44,149]$ & $\$ 2,740[\$ 0-\$ 34,442]$ & $\$ 2,583[\$ 0-\$ 34,442]$ & $\$ 2,771[\$ 0-\$ 91,816]$ \\
\hline \multirow[t]{2}{*}{ Pharmacy Cost ${ }^{* *} \dagger \neq$} & $\$ 4,361( \pm \$ 5,344)$ & $\$ 10,358( \pm \$ 5,657)$ & $\$ 13,472( \pm \$ 3,787)$ & $\$ 13,187( \pm \$ 3,935)$ & $\$ 4,757( \pm \$ 5,597)$ \\
\hline & $\begin{array}{l}\$ 3,445[\$ 829- \\
\$ 72,703]\end{array}$ & $\begin{array}{l}\$ 8,915[\$ 5,902- \\
\$ 40,300]\end{array}$ & $\begin{array}{c}\$ 12,301[\$ 9,130- \\
\$ 34,737]\end{array}$ & $\begin{array}{c}\$ 12,080[\$ 5,839- \\
\$ 34,737]\end{array}$ & $\begin{array}{l}\$ 3,567[\$ 829- \\
\quad \$ 72,703]\end{array}$ \\
\hline
\end{tabular}

* Proportion of days covered.

**Mean, standard deviation, median, [range].

† ANOVA pairwise comparison between PDC $<50 \%$ and PDC $\geq 80 \%$ groups, $p<0.05$.

$\neq t$-test between 'Persistent' and 'Non-Persistent' (60 Day Gap) groups, $\mathrm{p}<0.05$.

$\triangle$ Chi-square comparison between PDC $<50 \%$ and PDC $>80 \%$ groups, $\mathrm{p}<0.05$.

$¥$ Chi-square comparison between 'Persistent' and 'Non-Persistent' (60 Day Gap) groups, $p<0.05$. 
Table 6 Teriparatide utilization and fracture-related utilization and costs in the 12 month follow-up period

\begin{tabular}{|c|c|c|c|c|c|}
\hline Measure & $\begin{array}{l}\mathrm{PDC}^{*}<50 \% \\
\text { low adherent }\end{array}$ & $\begin{array}{c}\text { PDC } 50 \% \leq \mathbf{8 0} \% \\
\text { moderately } \\
\text { adherent }\end{array}$ & $\begin{array}{c}\text { PDC } \geq 80 \% \\
\text { highly adherent }\end{array}$ & Persistent & $\begin{array}{l}\text { Non-perstistent } \\
\text { (60 day gap) }\end{array}$ \\
\hline Total Patients, n (\%) & $168(75.7 \%)$ & $15(6.8 \%)$ & $39(17.6 \%)$ & $43(19.4 \%)$ & $179(80.6 \%)$ \\
\hline \multicolumn{6}{|c|}{ Fracture-Related Inpatient Hospitalization } \\
\hline $\begin{array}{l}\text { Members with Hospitalization, } \\
\text { n (\%) }\end{array}$ & $18(10.7 \%)$ & $1(6.7 \%)$ & $2(5.1 \%)$ & $2(4.7 \%)$ & $19(10.6 \%)$ \\
\hline \multirow[t]{2}{*}{ Hospitalizations Per Member** } & $0.1( \pm 0.4)$ & $0.1( \pm 0.3)$ & $0.1( \pm 0.2)$ & $0 .( \pm 0.2)$ & $0.1( \pm 0.4)$ \\
\hline & $0[0-2]$ & $0[0-1]$ & $0[0-1]$ & $0[0-1]$ & $0[0-2]$ \\
\hline \multicolumn{6}{|l|}{ Fracture-Related Emergency Room Visits } \\
\hline Members with Visit, n (\%) & $31(18.5 \%)$ & $1(6.7 \%)$ & $3(7.7 \%)$ & $3(6.98 \%)$ & $32(17.9 \%)$ \\
\hline \multirow[t]{2}{*}{ Visits Per Member** } & $0.2( \pm 0.5)$ & $0.1( \pm 0.3)$ & $0.1( \pm 0.5)$ & $0.1( \pm 0.5)$ & $0.2( \pm 0.5)$ \\
\hline & $0[0-2]$ & $0[0-1]$ & $0[0-3]$ & $0[0-3]$ & $0[0-2]$ \\
\hline \multicolumn{6}{|l|}{ Fracture-Related Outpatient Visits } \\
\hline Members with Visit, n (\%) & $160(95.2 \%)$ & 15 (100.\%) & $38(97.4 \%)$ & $42(97.7 \%)$ & $171(95.5 \%)$ \\
\hline \multirow[t]{2}{*}{ Visits Per Member** } & $4.2( \pm 7.96)$ & $4.3( \pm 5.14)$ & $3.8( \pm 5.48)$ & $3.4( \pm 5.21)$ & $4.3( \pm 7.83)$ \\
\hline & $2[0-65]$ & $2[1-17]$ & $2[0-32]$ & $2[0-32]$ & $2[0-65]$ \\
\hline \multicolumn{6}{|l|}{ Fracture-Related Pharmacy Claims } \\
\hline \multicolumn{6}{|l|}{ Pain Medications } \\
\hline $\begin{array}{l}\text { Members with Pharmacy } \\
\text { Claim, } \mathrm{n}(\%)\end{array}$ & $126(75.0 \%)$ & $11(73.33 \%)$ & $24(61.54 \%)$ & $27(62.79 \%)$ & $134(74.86 \%)$ \\
\hline \multirow{2}{*}{$\begin{array}{l}\text { Pharmacy Claims Per } \\
\text { Member** }\end{array}$} & $5.5( \pm 7.3)$ & $6.1( \pm 10.2)$ & $5.7( \pm 8.4)$ & $5.5( \pm 8.4)$ & $5.6( \pm 7.5)$ \\
\hline & $2[0-43]$ & $2[0-33]$ & $2[0-42]$ & $2[0-42]$ & $2[0-43]$ \\
\hline \multicolumn{6}{|l|}{ Osteoporosis Medications } \\
\hline $\begin{array}{l}\text { Members with Pharmacy Claim, } \\
\text { n (\%) }\end{array}$ & $168(100 . \%)$ & $15(100 \%)$ & $39(100 . \%)$ & $43(100 . \%)$ & $179(100 . \%)$ \\
\hline \multirow[t]{2}{*}{ Pharmacy Claims Per Member ${ }^{* *} \dagger \neq$} & 4. $( \pm 3.3)$ & 9. $( \pm 1.4)$ & $12.5( \pm 2.4)$ & $12.2( \pm 2.5)$ & $4.3( \pm 3.4)$ \\
\hline & $3[1-16]$ & $9[7-12]$ & $13[5-18]$ & $12[5-18]$ & $3[1-16]$ \\
\hline
\end{tabular}

Total Healthcare Cost ${ }^{* *} \dagger \neq$

$\$ 4,419( \pm \$ 6,018)$
$\$ 2,710[\$ 854-$
$\$ 40,637]$

$\$ 9,292( \pm \$ 4,396)$

$\$ 8,111[\$ 5,850-$ $\$ 23,884]$

Inpatient Hospitalization Cost**

$\$ 1,325( \pm \$ 4,988)$

$\$ 830( \pm \$ 3,214)$

$\$[\$ 0-\$ 37,355]$

$\$[\$ 0-\$ 12,449]$

$\$ 44( \pm \$ 132)$

$\$ 9( \pm \$ 36)$

$\$ 0[\$ 0-\$ 753]$

$\$ 0[\$ 0-\$ 141]$

Outpatient Cost ${ }^{* *}$

$\$ 767( \pm \$ 1,903)$
$\$ 159[\$ 0-\$ 12,380]$

$\$ 955( \pm \$ 1,538)$

$\$ 220[\$ 0-\$ 5,733]$

$\$ 1,048( \pm \$ 3,716)$

$\$ 215[\$ 0-\$ 23,383]$

$\$ 2,068( \pm \$ 1,196)$

Osteoporosis Medication Cost ${ }^{* *} \dagger \neq$

\section{$\$ 12,199( \pm \$ 4,932) \quad \$ 4,756( \pm \$ 6,099)$ \\ $\$ 11,263[\$ 7,616-\quad \$ 2,765[\$ 854-$ $\$ 36,026]$ \\ $\$ 40,637]$}

$$
\begin{array}{cc}
\$ 332( \pm \$ 1,930) & \$ 1,313( \pm \$ 4,913) \\
\$[\$ 0-\$ 12,585] & \$[\$ 0-\$ 37,355] \\
\$ 6( \pm \$ 23) & \$ 42( \pm \$ 129) \\
\$ 0[\$ 0-\$ 128] & \$ 0[\$ 0-\$ 753] \\
& \\
\$ 941( \pm \$ 3,545) & \$ 802( \pm \$ 1,892) \\
\$ 186[\$ 0-\$ 23,383] & \$ 176[\$ 0-\$ 12,380] \\
& \\
\$ 10,516( \pm \$ 1,520) & \$ 2,391( \pm \$ 1,746) \\
\$ 10,748[\$ 6,836- & \$ 1,735[\$ 779- \\
\$ 14,910] & \$ 9,631]
\end{array}
$$
$\$ 1,713[\$ 779$ - $\$ 5,259]$

\section{$\$ 7,420( \pm \$ 1,169)$ \\ $\$ 7,350[\$ 5,744-$} $\$ 9,631]$
$\$ 10,810( \pm \$ 1,250)$

$\$ 10,969[\$ 8,811-$ $\$ 14,910]$ 


\begin{tabular}{|c|c|c|c|c|c|}
\hline \multirow[t]{2}{*}{ Pain Medication Pharmacy Cost** } & $\$ 216( \pm \$ 646)$ & $\$ 77( \pm \$ 172)$ & $\$ 440( \pm \$ 1,201)$ & $\$ 404( \pm \$ 1,150)$ & $\$ 208( \pm \$ 628)$ \\
\hline & $\$ 28[\$ 0-\$ 6,071]$ & $\$ 11[\$ 0-\$ 662]$ & $\$ 18[\$ 0-\$ 6,276]$ & $\$ 14[\$ 0-\$ 6,276]$ & $\$ 27[\$ 0-\$ 6,071]$ \\
\hline
\end{tabular}

osteoporosis medication costs when low adherent patients were compared those who were highly-adherent, and when non-persistent members were compared with those who were persistent. An ANOVA pair-wise comparison revealed that the members with low adherence had, on average, significantly lower total costs $(M=\$ 4,419, \mathrm{SD}=$ $\$ 6,018)$, than did members with high adherence $(M=$ $\$ 12,670, \mathrm{SD}=\$ 4,963 \mathrm{~F}=63.254 \mathrm{p}<0.0001)$. In addition, t-test results showed significantly lower total costs among non-persistent members $(\mathrm{M}=\$ 4,756, \mathrm{SD}=\$ 6,099)$, as compared to the costs of persistent members $(\mathrm{M}=$ $\$ 12,199, \mathrm{SD}=\$ 4,932 \mathrm{t}=7.44, \mathrm{p}<0.0001)$.

With regard to osteoporosis medication costs, the average costs during the follow up period were significantly lower for members demonstrating low adherence $(M=\$ 2,068$, $\mathrm{SD}=\$ 1,196)$, as compared to the higher costs for those with high adherence $(M=\$ 10,810, S D=\$ 1,250 F=1662.62$ $\mathrm{p}<0.0001)$. In addition, there were significant differences in osteoporosis medication costs when comparisons were made between members who were non-persistent and members who were persistent. These costs were significantly lower for non-persistent members $(M=\$ 2,391$, SD $=\$ 1,746)$ than for persistent members $(M=\$ 10,516, S D$ $\$ 1,520 \mathrm{t}=28.06 \mathrm{p}<0.0001)$.

\section{Association between teriparatide adherence and teriparatide persistence with fracture rates}

Multivariate logistic regression analyses were used to assess the associations between adherence and persistence and the risk for a fracture in the 12-month period following teriparatide initiation. As noted in Figures 2 and 3, two separate models were evaluated. The results in Figure 2 describe the association of adherence and fracture outcome, and those in Figure 3 depict the association of persistence and fracture outcome. Age, gender, geographic region, race/ethnicity, prior osteoporosis medication use, Deyo-Charlson comorbidity, and prior fracture were covariates in both models.

As noted in Figures 2 and 3 and in Table 7, there were no significant differences in fracture outcomes whether or not patients were persistent in taking teriparatide (OR 1.2, 95\% CI $0.79,1.82$, NS), or regardless of their adherence status (high versus low adherence [OR .81, 95\% CI 0.515- 1.231, NS], and moderate versus low adherence [OR .91.95\% CI 0.479, 1.741, NS]). While the regression models demonstrated no significant association between teriparatide adherence or persistence and 12-month fracture outcomes, the results indicated that patients who had fractures in the 6 months before initiation of TPTD were almost 3 times more likely to have a fracture in the follow-up period (OR 2.9, 95\% CI 2.1-4.1 p $<0.0001)$. Furthermore, only $28 \%$ of members continued teriparatide use after their first fracture episode.

\section{Discussion}

This study explored the teriparatide utilization patterns of patients with Medicare Advantage with Prescription Drug (MAPD) coverage, excluding LIS beneficiaries, who initiated teriparatide therapy, and the manner in which those utilization patterns were associated with fracture outcomes and healthcare utilization. While the results suggested that there were no significant differences in fracture outcomes whether or not patients persisted with taking the drug or whether or they were adherent or not, this study identified specific patterns in utilization and cost in this population.

The results of the adherence component of this study support findings of other studies that have evaluated osteoporosis therapies. Similar to previous studies, these findings suggest that patients with osteoporosis tend to have poor adherence to or persistence with prescribed therapies, and that this poor adherence/persistence may be associated with adverse clinical and economic outcomes [24-27]. The results indicated that only $21 \%$ of the study group was optimally adherent (PDC $\geq 80 \%$ ) to their teriparatide therapy at one year of follow up, and that only $24 \%$ of patients continued to take the drug during that same time period. Some studies have reported higher rates of adherence with teriparatide therapy 12 to 18 months following therapy initiation as compared to this study's findings [28-30]. Arden and colleagues [29] reported an $87 \%$ persistence rate among 435 patients residing in the U.K., and Adachi et al [30] reported an $82 \%$ adherence rate in a cohort of 116 patients participating in an 18-month, multi-center prospective study of teriparatide adherence. In addition, a retrospective study by $\mathrm{Yu}$ et al [6] demonstrated an 


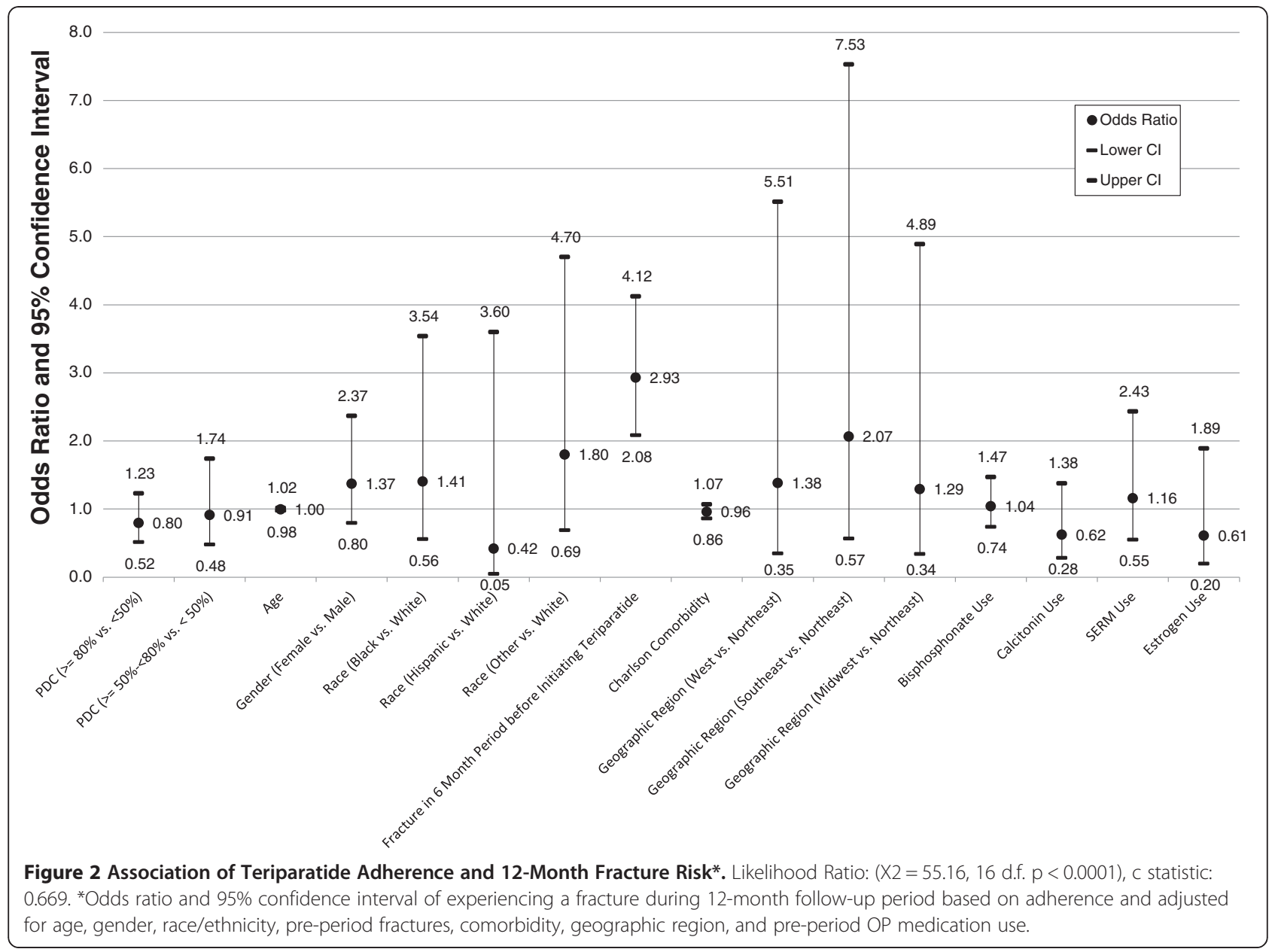

adherence rate of $81 \%$ in a cohort generated by the combined administrative claims of 3,587 commercial and Medicare patients in the U.S. The difference in these results may be due to the fact that the patient pool for this study was comprised solely of Non-LIS Medicare Part D beneficiaries, a large percentage of which had reached the coverage gap in the 12 months following initiation of teriparatide. The lack of funds to cover the cost of the medication once patients reach the coverage gap could explain the observed lower rates of adherence.

A surprising finding in this study was that less than half of the patient cohort (46.4\%) had used another osteoporosis medication in the 6 months prior to initiating teriparatide therapy. In lieu of choosing to take an oral medication once weekly or monthly, a large percentage of patients initiated a once-daily injectable dosage form (teriparatide). Teriparatide is recommended for patients at high risk of fracture, and so initiation in treatment naïve patients does occur; however, common clinical practice is for teriparatide to be utilized after treatment failure on first line therapy. An additional surprising finding is the low incidence of prevalent fracture (50\%). With teriparatide's indication for use, one would have expected a much higher incidence of patients with prevalent fracture. In clinical practice, teriparatide may often be utilized after treatment failure on another osteoporosis medication [8,31]. This study's analysis did not allow for the assessment of the level of severity of osteoporosis in the patients, which presumably would be more severe in this cohort based on the degree of initiation of teriparatide. It is possible that some health care providers may be prescribing teriparatide as a first line agent based on a comprehensive assessment of patients' disease severity.

Interestingly, there was a low rate of persistence with teriparatide after the first observed fracture. In fact, only $28 \%$ of patients in the study continued using the drug after the first fracture; however, the underlying cause for this non-persistence could not be investigated due to the claims based structure of this study. It was not clear whether the non-persistence was due to patient apathy toward the benefit of their therapy, to patients 
attributing the causes of their fractures to treatment failure, or due to prescribers deciding to discontinue the therapy and opting for another treatment strategy.

The analyses evaluating the relationship between utilization patterns (adherence or persistence) and fracture-related utilization and costs revealed interesting trends. The results suggested that patients who were highly adherent or persistent with teriparatide therapy had lower rates of emergency room utilization. among patients who had poor adherence or non-persistence with teriparatide therapy. Conversely, the patient groups that were highly-adherent and/or were persistent with teriparatide therapy had higher overall and pharmacy costs than did their non-adherent/non-persistent counterparts; almost $70 \%$ of the total costs of these highlyadherent and persistent patients were attributable to pharmacy expenses.

Our finding of a higher risk of fractures among patients who had experienced fractures in the 6 months prior to teriparatide initiation is consistent with previous studies of patients with osteoporosis [32-34]. Klotzbuecher and colleagues' review determined that patients with histories of previous fractures at different sites have a two-fold increased risk of having a future fracture [32]. Similarly, Haentjens and colleagues' meta-analysis suggested that prior wrist or spine fracture was associated with an increased risk of future hip fracture in both men and women [33]. Furthermore, Lindsay and colleagues' analysis of data from international trials of osteoporosis treatment estimated that among older women who had a vertebral fracture, 20\% would experience another vertebral fracture and $26 \%$ would incur a vertebral or non-vertebral fracture within 12 months $[34,35]$. Our observations contribute to this pool of information and support the need to closely monitor and treat patients who have fractures in order to mitigate the risk of future fracture, which is known to increase morbidity and mortality rates $[1,35,36]$. Further research is warranted in order to better understand and reduce future risk, and to assess the efficacy of interventions for reducing the incidence of fracture among highrisk patients.

The present study has limitations that warrant consideration when interpreting the findings. This is an observational, retrospective claims-based study whose results

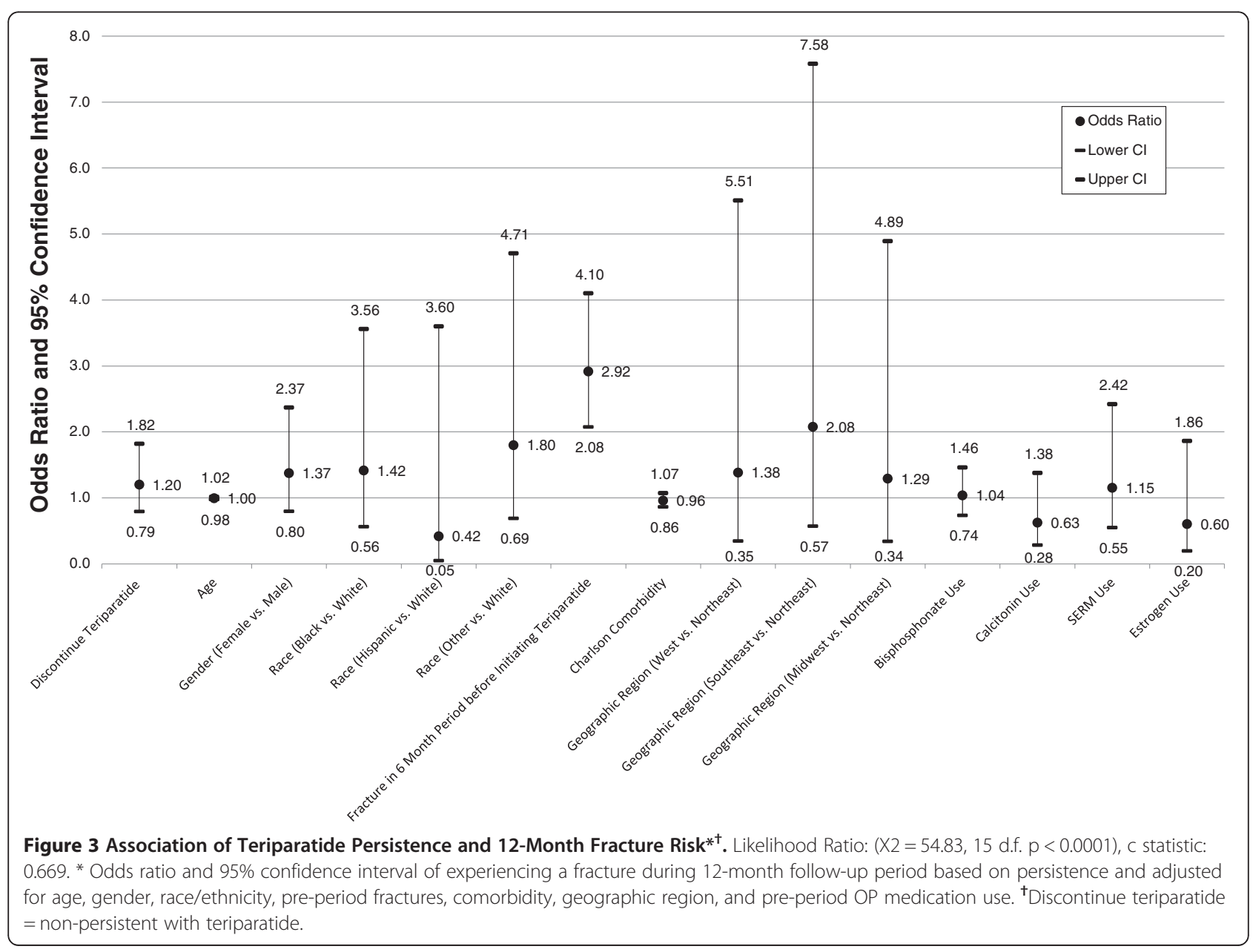


Table 7 Logistic regression of adherence and fracture risk* ${ }^{\dagger}$

\begin{tabular}{lccc}
\hline Variable & Odds ratio & 95\% confidence interval & P value \\
\hline PDC $(\geq 80 \%$ vs. $<50 \%)$ & 0.81 & $(0.515,1.231)$ & 0.48 \\
PDC $(\geq 50 \% \leq 80 \%$ vs. $<50 \%)$ & 0.91 & $(0.479,1.741)$ & 0.94 \\
Age & 1.00 & $(0.976,1.016)$ & 0.66 \\
Gender (Female vs. Male) & 1.37 & $(0.796,2.368)$ & 0.25 \\
Race (Black vs. White) & 1.41 & $(0.558,3.538)$ & 0.48 \\
Race (Hispanic vs. White) & 0.42 & $(0.048,3.599)$ & 0.29 \\
Race (Other vs. White) & 1.80 & $(0.689,4.703)$ & 0.22 \\
Fracture in 6 Month Period before Initiating Teriparatide & 2.93 & $(2.084,4.122)$ & $(0.861,1.07)$ \\
Charlson Comorbidity & 0.96 & $(0.347,5.513)$ \\
Geographic Region (West vs. Northeast) & 1.38 & $(0.566,7.531)$ & 0.46 \\
Geographic Region (Southeast vs. Northeast) & 2.07 & $(0.341,4.889)$ \\
Geographic Region (Midwest vs. Northeast) & 1.29 & $(0.738,1.469)$ \\
Bisphosphonate Use & 1.04 & $(0.283,1.378)$ \\
Calcitonin Use & 0.62 & $(0.551,2.433)$ & 0.05 \\
Selective Estrogen Receptor Modulator Use & 1.16 & $(0.197,1.891)$ \\
Estrogen Use & 0.61 & 0.82 \\
\hline
\end{tabular}

* Results of multivariate logistic regression analyses, with 'Fracture in 12 Month Post-Period' as the outcome variable.

${ }^{+}$Among patients with a fracture.

are indicative of associations of medication-use variables with clinical and economic outcome variables; the results do not show causal relationships among variables. In addition, other limitations common to studies using administrative claims data apply to this study. These include threats to validity presented by missing data, errors in claims coding, a lack of data on indirect costs, as well as unmeasured factors which may be associated with adherence. For example, the study did not control for potentially confounding factors such as patient health beliefs and provider-patient communication, which could have obscured the relationships of adherence and persistence with the clinical and economic outcomes. Additionally, the observations around prevalent fractures as well as use among treatment naïve patients makes it difficult to extrapolate these data to one better defined by the approved indication for use. Although multivariate regression modeling is used in the present study to reduce selection bias and control for confounding, it can only reduce bias caused by measured covariates; it cannot reduce bias caused by unmeasured covariates. Other study limitations may be the results of the criteria used to define the sample as well as those used to evaluate fracture outcomes. Patients who were LIS beneficiaries during the study period (560 patients) were excluded from the sample. Had those patients been included, the sample size would have been much larger, potentially increasing the generalizability of the study findings and permitting comparisons of the characteristics of the present study's patients with those of the LIS group. With regard to the assessment of fractures, the present study's analysis excluded fractures occurring within the first 90 days of teriparatide initiation, similar to approaches used in previous studies (e.g. 180 days used by Halpern et al. [27] so as to allow sufficient time for treatment effects to begin [37]) and assessed adherence and fracture outcomes concurrently. Some researchers posit that these approaches may give rise to inaccuracies in determining the association of adherence with fracture outcomes, and suggest that it is advisable to include an assessment of adherence prior to the occurrence of a fracture $[38,39]$. Finally, the current study uses data from a single large national health insurance company exclusively, thus the results might not be generalizable to the general U.S. population.

\section{Conclusions}

In summary, the study's findings suggest a need to better understand and improve adherence to teriparatide in Medicare Part D beneficiaries with osteoporosis. The results were inconclusive in terms of the exact relationship between poor adherence and negative outcomes such as increased utilization of ER visits and fracture rates. The results demonstrated a trend for highly-adherent patients to have higher healthcare costs most likely due to significantly higher pharmacy expenditures. Furthermore, patients who persisted with teriparatide use had significantly higher healthcare costs than patients who were nonpersistent with therapy. The results also suggested that emergency room utilization was higher among non- 
persistent patients and patients with low adherence; but that effect cannot be definitively tied to teriparatide use. In addition, the study's results highlight the importance of monitoring clinical outcomes of patients who have fractures in order to reduce the risk of future fractures. Future studies could evaluate the underlying factors contributing to higher healthcare utilization rates as well as the factors underlying more favorable outcomes such as lower utilization rates (for example, regression analyses with ER visits and inpatient hospitalizations as outcome variables).

\section{Methods}

\section{Study design}

This retrospective cohort study was designed to analyze the medical and pharmaceutical claims data of 761 Medicare members who had both pharmacy and medical benefits. The study's goals were to describe the clinical and economic outcomes of Medicare Part D beneficiaries newly started on teriparatide, and to examine the relationships of adherence and persistence with healthcare resource use and fracture outcomes. The data sources for this study included member enrollment, medical, and pharmacy data generated from the claims database of a large national healthcare provider. This study was part of a larger protocol, which was approved by the Western Institutional Review Board.

\section{Selection of participants}

The patient sample was drawn from a cohort of patients from a large national health insurance company who were enrolled in the Medicare Advantage with Prescription Drug coverage (MAPD) plan. In addition, patients included in the study were Medicare Part D recipients, aged 18 to 89 years old, and not members of the Low Income Subsidy program (LIS) for Medicare Part D at any time during the study period (LIS is a Medicare program which provides additional prescription drug coverage to low income Medicare Part D beneficiaries). LIS members were excluded from the study because they were more likely to be buffered from the cost-sharing effects of the Medicare Part D plan. Study participants had at least one pharmacy claim for teriparatide (Generic Product Identifier [GPI] code 30044070) during the identification period of January 1, 2008 to December 31, 2009. The date of the first pharmacy claim for teriparatide was considered the index date. In addition, participants had at least 18 months of total enrollment, that is, at least 6 months prior to the index date (baseline period) and at least 12 months post the index date (follow-up period). The patients' baseline demographics, pre-existing fracture rates, medication use, and comorbidities in the baseline period were evaluated. Clinical and economic outcomes were assessed during the 1-12 months postindex and the 1-24 months post-index.

\section{Primary outcome measures}

\section{Healthcare resource utilization}

The quantity of both inpatient and outpatient services received, as well as all-cause health care resource utilization in the claims data, were measured during the 12 and 24 month follow-up periods. This included outpatient (physician office visits, procedures and tests), inpatient hospitalizations, emergency room (ER) visits, and pharmaceutical claims.

\section{Healthcare costs}

Total overall costs and total fracture-related health care costs (reimbursements and co-pays) associated with the aforementioned health care resource utilizations, were measured during the follow-up period. All pharmacy and medical claims for members were examined during the follow-up period to assess total out of pocket pharmacy and medical spending. Costs were adjusted to 2010 dollars using the U.S. Bureau of Labor Statistics' Consumer Price Index for Medical Care. Fracturerelated services were characterized by an ICD-9 code according to the criteria used to identify fractures, which are outlined in the fracture section below.

\section{Fractures occurring in the 12 and 24-month follow-up period}

Fractures occurring in the follow-up period were assessed, with the exception of fractures occurring within the first 90 days following teriparatide initiation, as those fractures could not be reliably associated with osteoporosis treatment. The following criteria were used to identify the presence of fractures:

Fracture inclusion criteria Fractures were included if they were identified by the ICD-9-CM codes for fractures likely related to osteoporosis 813 to 814 (radius, ulna, and carpal fractures), 805 (vertebral fracture without spinal cord injury), 807.0 to 807.4 (rib fractures), 820 to 821 (femur fracture), or 808 to 809 (pelvic fracture and ill-defined fracture of the trunk). Also included were first fractures at any site identified by primary or secondary diagnosis (ICD 9) indicating a fracture on a non-diagnostic claim of inpatient, emergency room, or outpatient service, and ICD 9 codes (733.1) for pathologic fracture not related to cancer or Paget's disease.

Fracture exclusion criteria Fractures were excluded if they were identified by ICD 9 diagnosis codes associated with a malignant neoplasm (excluding non-melanoma skin cancer) or a benign tumor of bone in the 12 months before or in the 6 months following the date of the fracture, and if there was evidence for a malignancy that had diagnosis codes only for a history of cancer (ICD-9 V10). Pathological fractures were excluded if the 
patients were verified as having cancer within 6 months of the fracture diagnosis. Evidence of traumatic fracture (for example, fractures that were the result of a motor vehicular or pedal cycle accident), fractures accompanied by codes suggesting 3 or more simultaneous fractures, and open fractures, were excluded because they were likely to be the result of trauma rather than osteoporosis.

\section{Independent variables and covariates Covariates}

Age, gender and race/ethnicity Age, gender, and race/ ethnicity were captured from enrollment data. Patient age was measured as of the index date.

Geographic region Geographic region was based on the patient's state of residence on the index date. Regional assignment (Northeast, Midwest, South, and West) was based on the U.S. Census Bureau assignment of states to a geographic region.

Osteoporosis medication utilization This was evaluated using the pharmacy claims for each member during the baseline and follow-up periods. Utilization was based on receiving one or more paid pharmacy claims where the MediSpan(c) Generic Product Identifier (GPI) code was for a bisphosphonate (GPI-6 30.04.20), calcitonin (GPI-6 30.40.30), selective estrogen modulator (SERM) (GPI-8 30.05.30.60), or estrogen replacement therapy (GPI-4 24.99, 55.35).

The Deyo-Charlson comorbidity index (DCI) This index was used to assess the cohort's baseline comorbid conditions. It uses 17 categories of comorbidity to calculate a score that reflects cumulative increased likelihood of one-year mortality. It is based on ICD-9 diagnoses and procedure codes, and their associated weights. The DCI score can range from 0 to 33 . Claims with the specified codes are used in the calculation of the DCI if they meet the following criteria (1) Used on an inpatient hospitalization or (2) Two or more claims 30 days apart on separate outpatient claims [40].

Prior fracture Fractures episodes occurring in the 'pre' period (6 months before initiation of teriparatide) were also assessed using the aforementioned fracture inclusion/exclusion criteria for identification of fractures, described above.

\section{Independent variables}

Medication adherence - proportion of days covered (PDC) Medication adherence was calculated as the proportion of days covered (PDC). PDC was measured as a 12 month fixed follow-up period from the index date. The PDC was calculated as a percentage, which is equal to the number of days with teriparatide on hand divided by the number of days in the period. A PDC of 100 will equal 100\% adherence. The number of days with teriparatide on hand was calculated using a set of rules to avoid double-counting covered days when prescription fills for teriparatide overlap, as opposed to summing the day supply for all teriparatide prescriptions received during the period. Summing the days' supply is used in the calculation of a Medication Possession Ratio (MPR), another adherence measure. This method can overestimate the level of adherence during a fixed period of follow-up (i.e. 12 months, as in this study), especially if early refilling of prescriptions by the patient frequently occurs. 'Appropriate' or optimal medication usage was defined as having a PDC $\geq 80 \%$ and 'inappropriate' or suboptimal medication usage was defined as having a PDC of $<80 \%$. For the purpose of comparison, PDC was divided into the 3 categories of high, moderate and low. 'High' was defined as a PDC greater than or equal to $80 \%$ (PDC $\geq 80 \%$ ), 'moderate' was described as a PDC greater than or equal to $50 \%$ but less than $80 \%(50 \% \leq \mathrm{PDC}<80 \%)$, and 'low' was a PDC less than 50\% (PDC < 50\%).

\section{Therapy non-persistence}

Non-persistence with therapy (discontinuation) was defined as at least a 60-day gap in any subsequent teriparatide medication claim. The discontinuation date was defined as the date of the last prescription claim prior to the refill gap being exceeded, plus the day of supply of that claim. The refill gap was calculated using a procedure to avoid double-counting covered days when prescription fills for teriparatide overlap [41].

\section{Other variables \\ Pain medication utilization}

This was evaluated using the pharmacy claims for each member during the follow-up periods. Utilization was based on receiving one or more paid pharmacy claims where the GPI was for an opioid or non-steroidal anti-inflammatory drug (NSAID) therapy (GPI code 661000xx).

\section{Analysis plan}

A sample attrition table was produced to identify how many members were removed from the sample due to the specified exclusion/inclusion criteria. Descriptive statistics (mean, standard deviation and range for continuous variables, as well as percentages for dichotomous variables) were produced to summarize the group's demographic and clinical characteristics. Counts were provided (mean, median, standard deviation, and range) to summarize the overall and osteoporosis-related resource utilization and cost patterns for members with at least 12 months of post- 
period follow-up (i.e., months $1-12$ post index). The same measures were calculated for members with 24-months of post-period follow-up (i.e., months 1-24, post index). Similar counts were produced and then grouped according to patients' PDC status for comparison of 12 and 24 month outcomes. PDC calculations were used as predictor variables in analyses of 12 and 24 month clinical and economic outcomes. We summarized the 12 and 24 month overall and osteoporosis-related resource utilization and cost patterns between the groups of members based on their utilization of teriparatide. Chi Square analyses and ANOVA pairwise comparisons were performed to determine whether there were significant differences in healthcare utilization and costs for patients with high adherence (PDC $280 \%$ ) as compared to those with low adherence (PDC < $50 \%)$. T-Tests were used to assess significant differences in utilization and costs for patients who were persistent with therapy as compared to those who were non-persistent.

Multivariate logistic regression analyses were used to assess the association between adherence/persistence and fracture outcomes. Two regression models were evaluated to separately assess the associations of a) adherence and b) persistence, on fracture occurrence at follow-up. In the first model, adherence status was divided into the categories of high versus low ( $\mathrm{PDC}<80 \%$ vs. $\mathrm{PDC}<50 \%$ ), or moderate versus low $(50 \% \leq \mathrm{PDC}<80 \%$ vs. $\mathrm{PDC}<50 \%)$. For the second model, persistence with teriparatide was measured as dichotomous variable (non-persistence with treatment or persistence). In both models the dependent variable was the occurrence of a fracture during the 12-month follow-up period. Age, gender, geographic region, race/ethnicity, prior osteoporosis medication use, Deyo-Charlson comorbidity, and prior fracture were included as covariates in the models. All analyses were performed using SAS Enterprise Guide 4.2 statistical software (Cary, North Carolina, USA). The significance level was set at $<0.05$ for all statistical analyses.

\section{Competing interests}

LHF, AML and CLU are employees of Competitive Health Analytics, Humana Inc., which received funding from Eli Lilly and Company to conduct this study. SAF and RTB are employees and stockholders of Eli Lilly and Company which provided funding for this study and is the manufacturer of teriparatide.

\section{Authors' contributions}

LHF drafted the manuscript. AML collected the data and performed the statistical analyses. SAF and RTB came up with the concept of the study. LHF, AML, CLU, SAF and RTB participated in the design of the study, interpretation of the results, and in the progress and critical revisions of the manuscript. All authors read and approved the final draft.

\section{Acknowledgements}

This study was sponsored by Eli Lilly and Company. The authors wish to thank Yunping Zhou, Mary Costantino, and Loran Smith of Competitive Health Analytics, Humana, for their critical reviews of earlier drafts of this manuscript.

\section{Author details}

'Competitive Health Analytics Inc., Humana Inc, 3501 SW 160 Ave, Miramar, FL 33029, USA. ${ }^{2}$ Eli Lilly and Company, Indianapolis, IN, USA.

Received: 18 May 2012 Accepted: 27 December 2012

Published: 3 January 2013

\section{References}

1. U.S. Department of Health and Human Services: Bone health and osteoporosis: a report of the surgeon general. Rockville, MD: U.S. Department of Health and Human Services, Office of the Surgeon General; 2004.

2. Dontas IA, Yiannakopoulos CK: Risk factors and prevention of osteoporosis-related fractures. J Musculoskelet Neuronal Interact 2007, 7:268-272.

3. Sandhu S, Hampson G: The pathogenesis, diagnosis, investigation and management of osteoporosis. J Clin Pathol 2011, 11:1042e-1050e.

4. Chesnut $\mathrm{CH}$ : Treating osteoporosis with bisphosphonates and addressing adherence: a review of oral ibandronate. Drugs 2006, 66:1351-1359.

5. Bock O, Felsenberg D: Bisphosphonates in the management of postmenopausal osteoporosis - optimizing efficacy on clinical practice. Clin Interv Aging 2008, 3:279-297.

6. Yu S, Burge RT, Foster SA, Gelwicks S, Meadows ES: The impact of teriparatide adherence and persistence on fracture outcomes. Osteoporos Int 2012, 23:1103-1113.

7. Downey TW, Foltz S, Boccuzzi SJ, Omar MA, Kahler KH: Adherence and persistence associated with the pharmacologic treatment of osteoporosis in a managed care setting. South Med J 2006, 99:570-575.

8. Kennel KA, Drake MT: Adverse effects of bisphosphonates: implications for osteoporosis management. Mayo Clin Proc 2009, 84:632-638.

9. Lee $S$, Glendenning P, Inderjeeth CA: Efficacy, side effects and route of administration are more important than frequency of dosing of antiosteoporosis treatments in determining patient adherence: a critical review of published articles from 1970 to 2009. Osteoporos Int 2011, 22:741-753.

10. Hodsman AB, Bauer DC, Dempster DW, Dian L, Hanley DA, Harris ST, Kendler DL, McClung MR, Miller PD, Olszynski WP, Orwoll E, Yuen CK: Parathyroid hormone and teriparatide for the treatment of osteoporosis: a review of the evidence and suggested guidelines for its use. Endoc Rev 2005, 26:688-703.

11. Gold DT, Weinstein DL, Pohl G, Krohn KD, Chen Y, Meadows ES: Factors associated with persistence with teriparatide therapy: results from the DANCE observational study. J Osteoporos 2011, :314970. doi:10.4061/2011/ 314970.

12. Tamariz L, Uribe CL, Luo J, Hanna JW, Ball DE, Krohn K, Meadows ES: Persistence with biologic therapies in the medicare coverage gap. Am J Manag Care 2011, 17:753-759.

13. Raebel M, Delate T, Ellis JL, Bayliss EA: Effects of reaching the drug benefit threshold on medicare members' healthcare utilization during the first year of Medicare Part D. Med Care 2008, 46:1116-1122.

14. Hsu J, Fung V, Price M, Huang J, Brand R, Hui R, Fireman B, Newhouse JP: Medicare Beneficiaries' knowledge of Part D prescription drug program benefits and responses to drug costs. JAMA 2008, 226:1929-1936.

15. Patel UD, Davis MM: Falling into the doughnut hole: drug spending among beneficiaries with end-stage renal disease under Medicare pPart D plans. J Am Soc Nephrol 2006, 17:2546-2553.

16. Madden JM, Graves AJ, Zhang F, Adams AS, Briesacher BA, Ross-Degnan D, Gurwitz JH, Pierre-Jacques M, Gelb Safran D, Adler GS, Soumerai SB: Costrelated medication nonadherence and spending on basic needs following implementation of Medicare Part D. JAMA 2008, 299:1922-1928.

17. Zhang JX, Yin W, Sun SX, Alexander GC: The impact of Medicare Part D prescription benefit on generic drug Use. J Gen Intern Med 2008, 23:1673-1678.

18. Tseng CW, Brook RH, Keeler E, Steers WN, Mangione CM: Cost-lowering strategies used by Medicare beneficiaries who exceed drug benefit caps and have a gap in drug coverage. JAMA 2004, 292:952-960.

19. Cronk A, Humphries TL, Delat T, Clark D, Morris B: Medication strategies used by Medicare beneficiaries who reach the Part $D$ standard drugbenefit threshold. Am J Health Syst Pharm 2008, 65:1062-1070.

20. Conwell L, Esposito D, Garavaglia S, Meadows ES, Colby M, Herrera V, Goldfarb S, Ball D, Marciniak M: Out-of-pocket drug costs and drug 
utilization patterns of postmenopausal Medicare beneficiaries with osteoporosis. Am J Geriatr Pharmacother 2011, 9:241-249.

21. Gandek B, Sinclair SJ, Kosinski M, Ware JE: Psychometric evaluation of the SF- $36^{\circledR}$ health survey in medicare managed care. Health Care Financ Rev 2004, 25:5-25

22. Polinski JM, Bhandari A, Saya UY, Schneeweiss S, Shrank WH: Medicare beneficiaries' knowledge of and choices regarding Part D, 2005 to the present. J Am Geriatr Soc 2010, 58:950-966.

23. Brod M, Rousculp M, Cameron A: Understanding compliance issues for daily self-injectable treatment in ambulatory care settings. Patient Prefer Adherence 2008, 2:129-136

24. Brookhart MA, Avorn J, Katz JN, Finkelstein JS, Arnold M, Polinski JM, Patrick AR, Mogun H, Solmon DH: Gaps in treatment among users of osteoporosis medications: the dynamics of noncompliance. Am J Med 2007, 120:251-256.

25. Foster SA, Foley KA, Meadows ES, Johnston JA, Wang SS, Pohl GM, Long SR: Adherence and persistence with teriparatide among patients with commercial, Medicare, and Medicaid insurance. Osteoporos Int 2011, 22:551-557.

26. Gold DT: Medication adherence: a challenge for patients with postmenopausal osteoporosis and other chronic illnesses. J Manag Care Pharm 2006, 12(Suppl S-a):S20-S25.

27. Halpern R, Becker L, Iabal SU, Kazis LE, Macarios D, Badamgarav E: The association of adherence to osteoporosis therapies with fracture, allcause medical costs, and all-cause hospitalizations: a retrospective claims analysis of female health plan enrollees with osteoporosis. J Manag Care Pharm 2011, 17:25-39.

28. Fogelman I, Fordham JN, Fraser WD, Spector TD, Christiansen C, Morris SA, Fox J: Parathyroid hormone(1-84) treatment of postmenopausal women with Low Bone Mass Receiving Hormone Replacement Therapy. Calcif Tissue Int 2008, 83:85-92.

29. Arden NK, Earl S, Fisher DJ, Cooper C, Carruthers S, Goater M: Persistence with teriparatide in patients with osteoporosis: the UK experience. Osteoporos Int 2006, 7:1626-16299.

30. Adachi JD, Hanley DA, Lorraine JK, Yu M: Assessing compliance, acceptance, and tolerability of teriparatide in patients with osteoporosis who fractured while on antiresorptive treatment or were intolerant to previous antiresorptive treatment: an 18-month, multicenter, open-label, prospective study. Clin Ther 2007, 29:2055-2067.

31. Sikon A, Batur P: Profile of teriparatide in the management of postmenopausal osteoporosis. Int J Womens Health 2010, 2:37-44.

32. Klotzbuecher CM, Ross PD, Landsman PB, Abbott TA 3rd, Berger M: Patients with prior fractures have an increased risk of future fractures: $A$ summary of the literature and statistical synthesis. J Bone Miner Res 2000, 5:721-739.

33. Haentjens $P$, Autier $P$, Collins J, Velkeniers $B$, Vanderschueren D, Boonen S: Colles fracture, spine fracture, and subsequent risk of hip fracture in men and women. A meta-analysis. J Bone Joint Surg Am 2003, 85-A:1936-1943.

34. Lindsay R, Silverman SL, Cooper C, Hanley DA, Barton I, Broy SB, Licata A, Benhamou L, Geusens P, Flowers K, Stracke H, Seeman E: Risk of new vertebral fracture in the year following a fracture. JAMA 2001, 285:320-323.

35. Lindsay R, Burge RT, Strauss DM: One year outcomes and costs following a vertebral fracture. Osteoporos Int 2005, 16:78-85.

36. Burge R, Dawson-Hughes B, Solomon DH, Wong JB, King A, Tosteson A: Incidence and economic burden of osteoporosis related fractures in the United States, 2005-2025. J Bone Miner Res 2007, 22:465-475.

37. Cummings SR, Black DM, Thompson DE, Applegate WB, Barrett-Connor E, Musliner TA, Palermo L, Prineas R, Rubin SM, Scott JC, Vogt T, Wallace R, Yates AJ, LaCroix AZ: Effect of alendronate on risk of fracture in women with low bone density but without vertebral fractures: results from the Fracture Intervention Trial. JAMA 1998, 280:2077-2082.

38. Curtis JR, Westfall AO, Cheng H, Lyles K, Saag KG, Delzell E: Benefit of adherence with bisphosphonates depends on age and fracture type: Results from an analysis of 101,038 new bisphosphonate users. J Bone Miner Res 2008, 23:1435-1441.

39. Siris ES, Harris ST, Rosen CJ, Barr CE, Arvesen JN, Abbott TA, Silverman S: Adherence to bisphosphonate therapy and fracture rates in osteoporotic women: relationship to vertebral and nonvertebral fractures from 2 US claims databases. Mayo Clinic Proc 2006, 81:1013-1022.
40. Deyo RA, Cherkin DC, Ciol MA: Adapting a clinical comorbidity index for use with ICD-9-CM administrative databases. Clin Epidemiol 1992, 45:613-619.

41. Leslie RS: Using Arrays to Calculate Medication Utilization. Orlando, FL: Proceedings of the 2007 SAS Global Forum; Paper 043-2007. http://www. lexjansen.com/pharmasug/2008/pr/pr07.pdf.

doi:10.1186/1471-2474-14-4

Cite this article as: Hazel-Fernandez et al: Association of teriparatide adherence and persistence with clinical and economic outcomes in Medicare Part D recipients: a retrospective cohort study. BMC

Musculoskeletal Disorders 2013 14:4.

\section{Submit your next manuscript to BioMed Central and take full advantage of:}

- Convenient online submission

- Thorough peer review

- No space constraints or color figure charges

- Immediate publication on acceptance

- Inclusion in PubMed, CAS, Scopus and Google Scholar

- Research which is freely available for redistribution

Submit your manuscript at www.biomedcentral.com/submit
C Biomed Central 